\title{
El Programa de Trabajadores Agrícolas Mexicanos con Canadá: un contraste frente a la experiencia con Estados Unidos
}

\section{Gustavo Verduzco Igartúa*}

El objetivo de este artículo es presentar las características principales de un programa internacional de trabajadores que lleva ya más de 25 en operación, aunque hasta ahora ha sido poco conocido. En el articulo se analizan particularmente las características contextuales de los cambios económicos que han ocurrido en Canadá y que han tenido como consecuencia la necesidad de complementar sus necesidades laborales estacionales con trabajadores temporales del Caribe y de México. Por otra parte, en la literatura sobre las migraciones laborales ha sido más frecuente la estigmatización de los programas de trabajadores "huéspedes" al imputarles problemas diversos en los países de destino; sin embargo, la presentación de los diversos datos del programa con Canadá nos permiten ver que éste ha sido eficazmente complementario de ciertas necesidades laborales agricolas de aquel país, sin que hasta ahora, después de tantos años, se perciban problemas que demeriten los logros del mismo.

\section{Introducción}

Hace 25 años que los gobiernos de Canadá y México firmaron un acuerdo que permitiría que trabajadores mexicanos fueran a Canadá a trabajar temporalmente en la agricultura de aquel país. ${ }^{1}$ Para México esta experiencia no era nueva ya que en el pasado había tenido lugar un programa similar con Estados Unidos, sólo que mucho mayor.

En 1942 debido al aumento del costo de los salarios en la agricultura estadunidense dentro del contexto de la segunda guerra mundial, México firmó un acuerdo con el país vecino mediante el que se permitiría el ingreso temporal a trabajadores mexicanos con el objeto de trabajar en labores agrícolas especialmente en el estado de $\mathrm{Ca}$ -

* Profesor-investigador de El Colegio de México.

El autor agradece a Daniel Díaz, funcionario de la Secretaría del Trabajo y Previsión Social, las informaciones y el apoyo que le proporcionó para conocer el programa de trabajadores agrícolas con Canadá. Asimismo agradece el apoyo recibido por parte del Conacyt.

${ }^{1}$ En términos oficiales fue un "Memorándum de entendimiento". 
lifornia. Fue éste un acuerdo que duró 22 años para culminar finalmente en medio de fricciones entre los dos gobiernos en 1964.

Son muchos y muy diversos los hechos y las circunstancias que tuvieron lugar entre 1942 y 1964 que explican tanto el inicio de tal programa, como su terminación conflictiva, y no sería objeto de este trabajo hablar de ello. Por lo pronto sólo cabe mencionar que a la par que se fue desarrollando el Programa Bracero, también fue creciendo un flujo continuo de migrantes indocumentados y, como es bien sabido, la migración mexicana indocumentada hacia Estados Unidos continúa hasta ahora como un punto de conflicto entre los dos países a pesar de su aparente funcionalidad como una respuesta a las fuerzas de la oferta y la demanda. En cambio el programa de trabajadores mexicanos agrícolas que México tiene con Canadá parece haberse desarrollado aparentemente bien y sin fricciones entre los dos gobiernos después de veinticinco años de existencia.

Hasta ahora, sin embargo, es muy poco lo que se ha sabido acerca de este programa más allá de algunas referencias generales que casi por casualidad han aparecido en la prensa o en alguna reunión pública o académica a pesar de haber transcurrido un largo cuarto de siglo a partir de esas experiencias. No parece ijustificable haber dejado de lado, desde el punto de vista del interés analítico, a un programa de este tipo y más aún si consideramos que ha operado sin interrupción por tanto tiempo. De ahí que la intención central de este trabajo es la de dar cuenta de varias de las circunstancias y características del programa a fin de poder reflexionar sobre ellas en el marco del análisis de los procesos migratorios.

Comúnmente se afirma, en la literatura sobre las migraciones internacionales, que las diferencias salariales y de desarrollo entre los países de origen y de destino constituyen un importante factor de atracción de las migraciones desde los países menos desarrollados, sobre todo cuando junto con estas circunstancias se dan otras como la vecindad o la cercanía o los lazos diversos que facilitan el acercamiento entre los mismos; obviamente éste parece haber sido el caso entre México y Canadá aunque en este mismo tenor se piensa, sin embargo, que los programas de trabajadores temporales terminan siendo un instrumento tanto para impulsar los flujos indocumentados como para el fomento de las migraciones permanentes; por ello tienden a ser vistos más bien con cierta desconfianza, particularmente ahora cuando existe un cierto temor por parte de los gobiernos y del público de los países del "norte" por causa del incremento de las 
migraciones que proceden del "sur". ${ }^{2}$ Además, algunos han llegado a interpretar que las migraciones podrían llegar a ser procesos de "autoperpetuación" mediante las redes sociales de apoyo, de tal manera que, aun sin existir factores de demanda laboral, las "redes" mismas pasarían a actuar como mecanismos de atracción con lo que se aumentarían todavía más las cargas sociales en los países de destino (Durand y Massey, 1992).

Por lo anterior, si tenemos por un lado a México como un país de menor desarrollo y, por otro lado a Canadá, y si además consideramos que ha existido un programa específico de trabajadores agrícolas durante 25 años (duración mayor a la del Programa Bracero), las visiones anteriores, expresadas en la literatura sobre el tema, nos llevarían a pensar que probablemente habrían ocurrido ya algunos de los fenómenos señalados más arriba, especialmente el cambio de migrante temporal a permanente, la presencia de migrantes indocumentados, así como una consolidación de las "redes sociales" que, según alguna interpretación, conducirían hacia un proceso de "autoperpetuación" de las migraciones más allá de los fenómenos de oferta y demanda laborales.

Por ello, aparte de la importancia que tiene en sí llegar a conocer un programa de trabajadores que lleva ya tantos años sin conflictos aparentes, las consideraciones anteriores nos plantearían varias interrogantes que tienen que ver no sólo con las modalidades comunes del fenómeno migratorio sino también con las percepciones e interpretaciones que se tienen sobre el mismo.

Es cierto que a primera vista podría pensarse que la enorme distancia que hay entre Canadá y México sería un obstáculo que seguramente limitaría los flujos migratorios, sin embargo, hemos de considerar que existen trabajadores mexicanos (tanto documentados como no documentados) en lugares tan lejanos como los estados de Nueva York o de Washington en Estados Unidos, ambos limítrofes con la frontera canadiense. En ei valle de Yakima, del esta 1 . de Washington, por ejemplo, lugar de gran importancia para la producción estadunidense de manzanas, peras, cerezas y lúpulo, entre otros produc-

\footnotetext{
${ }^{2}$ En la experiencia europea, particularmente de Alemania, existen evidencias en este sentido a partir de lo que fueron los "programas de trabajadores-huéspedes" de los años sesenta (Hammar, 1985). En la actualidad se ha expresado con cierto temor que “...Europa está en estado de sitio" por causa de los flujos de migrantes presentes y potenciales (United Nations Economie Commission for Europe, 1994).
} 
tos, $93 \%$ de la fuerza laboral agrícola es nacida en México y muchos de ellos son además indocumentados (Kissam, García y Runsten, 1992). De ahí que se pueda sospechar que podría ser factible que los mexicanos también estuvieran llegando a Canadá de manera semejante (indocumentada) a como en otros tiempos llegaron llegando a California en un proceso paralelo al del Programa Bracero.

En tiempos del Programa Bracero, la migración mexicana a Estados Unidos se fue expandiendo a otros sectores de la economía e incluyó también a los migrantes indocumentados, los que poco a poco fueron aumentando en número hasta llegar a ser una proporción importante frente a los que se documentaban mediante el programa. Luego, unos años más tarde, el programa terminó en medio de fricciones, aunque no por ello cesó el flujo laboral que continuó apoyando las labores agrícolas particularmente en California. ${ }^{3}$

Aparte de las inquietudes anteriores, el caso del programa de trabajadores agrícolas con Canadá nos plantea diversos tipos de preguntas, algunas de las cuales tienen que ver con las características contextuales con las que se inició el programa; otras con las formas comunes de operación y, existirían además, inquietudes como las planteadas inmediatamente antes, que tendrían que ver con efectos o resultados no directamente esperados.

Con el objeto de resolver algunos de los cuestionamientos anteriores, el autor de este artículo viajó a la provincia de Ontario, a fin de recabar la información necesaria. Además de realizar consultas en la biblioteca de la Universidad de York en Toronto, el autor revisó tanto los acervos bibliográficos de las oficinas del Ministerio de Agricultura de la provincia de Ontario, como los del Ministerio de Recursos Humanos. También tuvo oportunidad de entrevistar a varios de los funcionarios públicos que han estado a cargo del programa, y a representantes de los granjeros. ${ }^{4}$

${ }^{3}$ El tema sobre las circunstancias del fin del Programa Bracero es un punto de debate; sabemos, sin embargo, que durante muchos años caminaron paralelamente tanto los flujos documentados como los indocumentados así como también que después de la terminación del programa los flujos migratorios no cesaron, aunque la mayoría de los trabajadores ingresaba de manera indocumentada.

${ }^{4}$ Quiero agradecer en primer lugar el amplio respaldo recibido por la Universidad de York, mediante el profesor Alan Simmons, así como del personal del Center for Research for Latin America and the Caribbean (Cerlac) de la misma universidad. Especialmente agradezco el apoyo constante de Liddi Gomes; también el desinteresado apoyo del cónsul de México en Toronto, señor Aguilera-Beteta y del encargado del programa por parte del gobierno de la provincia de Ontario, Henry Neuefeldt. 
A continuación se expondrán algunos de los resultados que responden a varias inquietudes planteadas; como se verá, unos temas pudieron ser abordados de una manera más completa que otros. Asimismo, existen varias dimensiones del programa que no pudieron ser exploradas de manera sistemática en esa ocasión, ya que hubieran requerido de otro tipo de enfoques, de recursos financieros y de tiempo; por ejemplo, las percepciones de los granjeros canadienses sobre los trabajadores mexicanos y su desempeño laboral, así como las percepciones de los habitantes de los pueblos donde se ubican las granjjas, y temas afines. En el lado de México, por otra parte, carecemos de una etnografía de por lo menos alguno de los pueblos donde habitan estos trabajadores que participan en el programa canadiense y tampoco contamos con algún tipo de estudios que nos permita ahondar en las características diversas de estos trabajadores. Esta clase de investigaciones prometen ciertamente ser de gran interés y será deseable que puedan llegar a realizarse en un futuro próximo.

\section{Los objetivos del trabajo}

A continuación voy a presentar, primero, un perfil general tanto sobre el desarrollo de la agricultura canadiense, como acerca de la dinámica demográfica y del empleo agrícola, ya que sólo así podremos entender debidamente el sentido y el alcance de un programa como el que ahora nos ocupa. Después haré una presentación sobre las características sobresalientes del programaijunto con una visión preliminar del desempeño del mismo, basado en las percepciones de aquellos funcionarios públicos y privados que tienen que ver con el programa tanto en Canadá como en México. Al final propongo algunas ref lexiones que surgen del análisis con el objeto de rescatar ciertas orientaciones que podrían sernos útiles en el marco de estudios sobre las migraciones internacionales.

\section{La dinámica de la agricultura canadiense}

Veamos primero algunos indicadores generales de lo que han sido los principales cambios de la economía global canadiense en el periodo de nuestro interés, de acuerdo con las cifras convencionales que están disponibles (véase el cuadro 1). 


\section{CUADRO 1}

Principales cambios en la economía global canadiense

\begin{tabular}{lrrrr}
\hline & 1950 & $\%$ & \multicolumn{1}{c}{1990} & $\%$ \\
\hline Población canadiense & 13712000 & & 26440000 & \\
PEA & 5198000 & 38.0 & 13681000 & 58.0 \\
Trabajan en la agricultura & 1066000 & 20.5 & 429000 & 3.1 \\
Otras industrias primarias & 182000 & 3.5 & 283000 & 2.0 \\
Manufactura & 1321000 & 25.4 & 2001000 & 14.6 \\
Construcción & 335000 & 6.4 & 778000 & 5.7 \\
Servicios & 2138000 & 41.0 & 9081000 & 66.3 \\
\hline
\end{tabular}

Fuente: Vogt et al. (1993:56).

Puede apreciarse que mientras la población total apenas llegó casi a duplicarse en el periodo, la población económicamente activa (PEA) más que se dobló en números absolutos debido no sólo a la dinámica demográfica interna, sino en parte también por las políticas migratorias que incentivaron durante algunos años la llegada de extranjeros en edad de trabajar (Norrie y Owram, 1991; Beaujot, 1988), así como por la mayor incorporación de la mujer a diversos trabajos (Foot, 1987; Vogt et al., 1993). Por otra parte, como lo señalan las cifras anteriores, el empleo en la agricultura cayó drásticamente de $20.5 \%$ de la PEA, a solamente $3.1 \%$, mientras el empleo en las manufacturas bajó en números relativos de 25.4 a 14.6\%. En cambio, el empleo en los servicios se incrementó enormemente tanto en números relativos como absolutos, ya que a semejanza de otras economías postindustriales, Canadá redujó también su PEA agrícola y aumentó fuertemente su base de servicios diversos. Estos cambios han tenido características muy específicas que convienen resaltar. El fundamental ha sido el de la industrialización de la agricultura que a lo largo de los últimos años se puso de manifiesto con el aumento significativo de la cantidad y la calidad de los productos alimenticios en general.

Asimismo, mientras que por un lado se reduce el número de predios agrícolas, se ha dado un aumento importante tanto en el tamaño promedio de los predios, como en el del capital invertido (Hay, 1992; Statistics Canada, 1992). Los cambios anteriores se han visto también reflejados en una disminución notable no sólo de la población que habita en las zonas rurales sino de quienes residen en 
las granjas mismas ya que actualmente muchos granjeros prefieren vivir en los pequeños pueblos y ciudades cercanas a sus granjas que en éstas (Hay, 1992). Por otra parte, el válor total de la producción agrícola se ha incrementado más de cuatro veces, aunque proporcionalmente al conjunto de la economía, ha bajado frente al valor de la producción en otros sectores.

Al lado de los profundos cambios que se han dado en el sector agrícola, de todas formas sigue predominando la fuerza de trabajo masculina sobre la femenina, aunque en términos relativos esta última se ha incrementado. Además, ha seguido avanzando el envejecimiento de la fuerza de trabajo agrícola con mayor impulso todavía que para el conjunto de la población canadiense (Andarawewa, 1970; Foot, 1987; Hay, 1992). La escolaridad, sin embargo, ha seguido incrementándose al paso del tiempo en ese sector aunque a un ritmo más bajo que para el conjunto de la población canadiense (Foot, 1987). Por otra parte, el trabajo no agrícola ha seguido actuando como un imán sobre todo para la población joven, tendencia que se ha visto apoyada además por una diferencia salarial que favorece al trabajo urbano (Vogt et al., 1993; Norrie y Owran, 1991).

En síntesis, hay ahora menos granjas pero éstas son más grandes, hay en ellas más inversión de capital y son ahora más productivas a pesar de requerir de un menor número de trabajadores que en años pasados. En contrapartida, sin embargo, sus propietarios o administradores son ahora más viejos aunque tienen también más años de escolaridad que en otras épocas.

Pero como es común en otras actividades, en la agricultura también se reflejan enormes cambios regionales cuando se comparan las diversas zonas del país. Tenemos así que mientras que para todo Canadá el número de predios agropecuarios o "granjas" descendió en $60 \%$ entre 1941 y 1986 , la reducción fue todavía mayor para las provincias del Atlántico y de Quebec y, contrastadamente menor para las provincias de Columbia Británica y Alberta, mientras que en las provincias de Ontario, Manitoba y Saskatchewan el descenso ha sido similar al de todo el país. Estos cambios se deben a un conjunto de situaciones variadas entre las que destacan los diversos tipos de granjas en cuanto a su dedicación y organización en las diferentes regiones, lo cual ha hecho que el impacto de las transformaciones sea también diferencial (Hay, 1992).

Pero desde otro punto de vista, tenemos que las provincias con mayor proporción de granjas entre los años 1986 y 1991 han sido 
Ontario (24.2\%), Saskatchewan $(21.4 \%)$ y Alberta (20.3\%). Sin embargo llama la atención que al lado de los profundos cambios que han tenido lugar en casi todas las regiones, la provincia de Ontario se haya mantenido con una extensión de cultivo semejante a lo largo de todo el periodo desde 1941 (Hay, 1992).

Como lo veremos a continuación, la provincia de Ontario no sólo sobresale en otros aspectos generales del comportamiento económico entre las demás provincias de Canadá, sino que en cierta manera su agricultura es también marcadamente diferente del conjunto. En términos globales, el producto nacional bruto (PNB) de Ontario es $39.2 \%$ del total canadiense, y en agricultura es $26 \%$. Sin embargo por lo que se refiere a la producción y agroindustria de verduras y frutas, el PNB de la provincia alcanza hasta 59.9\%, indicador que nos muestra la gran importancia que este subsector agrícola de Ontario tiene en el conjunto del país.

Ontario es líder nacional en la producción de los siguientes productos agropecuarios: soya, $100 \%$; tabaco, $89 \%$; maíz, $67 \%$; verduras, 48\%; manzanas, $42 \%$; flores e invernaderos, $51 \%$; productos lácteos, $35 \%$; ovejas, $57 \%$; ganado, $21 \%$. Puede verse, por tanto, que la especialidad agrícola de Ontario está sobre todo en la producción de soya, maíz, verduras y frutas, flores, tabaco y productos lácteos.

Otras provincias como Saskatchewan y Alberta dominan, por ejemplo, en la producción de trigo, forraje, heno, ganado y lana. Sin embargo no hay que olvidar que una cuarta parte del PNB agrícola canadiense está en la provincia de Ontario. ${ }^{5}$ Debido a la variedad y riqueza de sus productos agropecuarios, Ontario es a Canadá, en cierta manera y guardando las enormes proporciones, lo que California es a Estados Unidos.

Hasta aquí hemos visto un panorama del perfil general de la agricultura canadiense con algunos de los cambios principales que se han experimentado en ese sector; veamos ahora de qué manera ha evolucionado la fuerza de trabajo agrícola. Se mencionaba más arriba que de manera paralela al cambio global de la economía canadiense, se ha dado una drástica reducción de la fuerza de trabajo agrícola, aunque como contrapeso tuvo lugar también un proceso de

${ }^{5}$ Estos datos han sido consultados en los siguientes documentos: "1992, Agricultural Statistics for Ontario" (Ministry of Agriculture and Food, 1993); "Changes in Agriculture in Canada: Theorical Perspectives" (Basran, 1992); "Rural Canada in Transition: Trends and Developments" (Hay, 1992). 
mecanización y de tecnificación que junto con la mayor inversión de capital han producido un incremento notable de la eficiencia y de la producción agropecuaria.

Sin embargo, en éste como en otros temas, también la agricultura de Ontario se desvía de la situación general, sobre todo si atendemos a las necesidades de algunos de los cultivos especifícos de esta provincia. Por ejemplo, los administradores de los predios y sus familias constituían $72 \%$ de la fuerza agrícola de Ontario en 1986, frente a 28\% de trabajadores asalariados de los cuales la mitad sólo se contrataba temporalmente. Pero en los cultivos de tabaco y actividades hortícolas de la misma provincia, los trabajadores asalariados constituían $58 \%$ de la fuerza de trabajo y de éstos $41 \%$ eran trabajadores asalariados estacionales. ${ }^{6}$ Además, en la producción de invernadero el número de empleados pasó de 6.8 miles a 18.7 miles entre 1990 y 1992, y tanto la extensión bajo producción como el valor de la misma crecieron en 31 y $21 \%$ respectivamente en esos mismos años (Ministry of Agriculture and Food, 1993). De hecho este tipo de producción ha sido el más dinámico del sector en los últimos años.

Por lo expuesto hasta aquí, podemos inferir que la provincia de Ontario liderea la producción de estos productos en Canadá y por esa razón cualquier crisis en la satisfacción de sus requerimientos laborales cobra una mayor importancia.

\section{Tendencias del mercado laboral en la agricultura de Ontario}

A semejanza de lo que ocurrió en todo Canadá, la población agrícola de Ontario bajó drásticamente (en 45\%) principalmente entre 1961 y 1981, y el número de granjas también descendió (en 32\%), aunque la extensión cultivada permaneció más o menos igual hasta 1992. Quiere decir que este cambio se dio en beneficio de la ampliación de la extensión promedio de las granjas, o sea que hubo menos granjas pero éstas se hicieron más grandes (Hay, 1992; Ministry of Agriculture and Food, 1992 y 1993).

Aunque ya en $196135 \%$ de los granjeros reportaba que complementaba su ingreso con trabajos no agrícolas, para 1981 subió hasta $44 \%$ como uno de los efectos colaterales de la mayor mecanización y 
tecnificación agrícolas, en parte también por las presiones para complementar ingresos y debido a las mejores oportunidades que se fueron abriendo particularmente en el sector no agrícola. Por otra parte, en términos monetarios, ya en 1986 sólo $27 \%$ de los ingresos de los granjeros provenía de las actividades agropecuarias frente a $49 \%$ que se derivaba de actividades asalariadas no agrícolas (Ministry of Agriculture and Food, 1991).

La proporción de "trabajadores familiares sin pago" fue bajando como consecuencia de un conjunto de factores, ya sea porque empezaron a darse mejores oportunidades de ingreso fuera de la agricultura o también porque se fue reduciendo el número de miembros por familia en las granjas. Por otro lado, se incrementó muy sustancialmente el número y la proporción de los "trabajadores asalariados" que subió en $78 \%$ en el mismo periodo.

En términos globales se mantuvo más o menos igual el tamaño de la fuerza agrícola ya que mientras bajó el número de granjeros y el de sus familiares no asalariados, subió el de la fuerza laboral asalariada, circunstancia que es importante tener en cuenta por el tema que nos ocupa en este trabajo.

La composición por el sexo de la fuerza agrícola fue otro cambio importante, pues en 1981 las mujeres llegaron a ser 56\% del total de los trabajadores agrícolas (asalariados y no asalariados), situación que ha continuado similar hasta el presente.

Como puede observarse, se trata de transformaciones que subrayan la orientación de la agricultura hacia un perfil más corporativo empresarial, donde a la par que se incrementó la capitalización, aumentó también la participación del trabajo asalariado y la mayor participación de la mujer. Pero son cambios que a su vez han tenido que ver con la evolución del conjunto social que ha implicado una mayor apertura de las oportunidades laborales urbanas, con mejores sueldos que en el campo.

\section{Ontario y la producción agrícola regional}

En otros párrafos se mencionaba la riqueza y variedad agrícolas de Ontario. Geográficamente la provincia se ha dividido en cinco regiones: el sur, el oeste, el centro, el este y el norte. De estas zonas, tanto la del sur como la del oeste son las más productivas agricolamente. El sur da cuenta de cerca de $40 \%$ de las ventas totales de la producción 
agrícola de la provincia, a semejanza de la zona oeste (35\%), es decir, que entre las dos juntan $75 \%$. En cambio las zonas central y oriental apenas dan cuenta de $11 \%$ cada una, y el boscoso norte completa con un raquítico 3\% (Ministry of Agriculture and Food, 1993).

El sur de Ontario es la zona de mayor producción en los siguientes cultivos: trigo de invierno, maíz, soya, tabaco, flores y verduras. De estos dos últimos produce $72 \%$ de toda la provincia y sobresalen particularmente el jitomate, el pepino y la lechuga. Por lo que respecta a la producción de tabaco, más de la mitad se produce en un solo condado (Haldimand-Norfolk), pero recordemos además que Ontario produce $89 \%$ de todo el tabaco canadiense. El oeste de la provincia se especializa en cebada, granos diversos, heno y ganado, aunque también tiene producción importante de maíz, verduras y frutas. El este se especializa en la producción de heno y ganado (Ministry of Agriculture and Food, 1993).

Por el tipo de productos particulares de cada región, es claro que la mayor demanda laboral ocurre en el sur, seguida del oeste, ya que en esos lugares hay cultivos como el tabaco, las flores y verduras que, por sus propias carácterísticas, requieren de mano de obra de manera intensiva, particularmente en lás épocas de cosecha. De hecho, según datos de los años ochenta, $63 \%$ de las granjas que cultivan alguno o varios de los productos mencionados requería de mano de obra estacional. Sobresalen las granjas productoras de tabaco ya que casi todas $(88 \%)$ utilizaban asalariados estacionales, seguidas por las productoras de fruta donde se daba el mismo requerimiento en $62 \%$, mientras que -de manera constrastante-apenas $28 \%$ de las granjas que cultivaban los otros productos requerían del mismo tipo de trabajadores. ${ }^{7}$

Además de que en las granjas de tabaco y de frutas y verduras se utiliza más mano de obra estacional, en las primeras, $57 \%$ de toda la mano de obra asalariada era estacional y $38 \%$ en las segundas, situación que hace ver más claramente la importancia de este tipo de trabajadores para esos cultivos. Para 1991, sin embargo, los requerimientos globales de mano de obra estacional habían aumentado en la provincia ya que $43 \%$ de todas las granjas utilizó este tipo de fuerza de trabajo. Este aumento también se vio reflejado proporcionalmen-

${ }^{7}$ Estas tendencias de la demanda laboral se mantuvieron más o menos iguales entre 1970 y 1980 . Los datos de esos años se encuentran en un reporte elaborado por el Joint Ontario Agricultural Human Resources Planning Committee (1986). Desafortunadamente no existen datos similares que permitan comparar con fechas más recientes. 
te en los diferentes cultivos según sus propias características; por ejemplo, $80 \%$ de las granjas productoras de fruta y verdura utilizó fuerza de trabajo estacional mientras que las granjas que cultivaron otros productos no intensivos, tuvieron una participación de $23 \%$, o sea un poco más baja que diez años antes como efecto de los procesos de mecanización que se han adaptado mejor a estos últimos cultivos (Statistics Canada, 1993). Pero referidos en particular a las llamadas "industrias de invernadero", el número total de trabajadores (de tiempo parcial y completo), subió entre 1990 y 1992 de 6842 a 18 777, o sea que tuvo un incremento de $274 \%$; pero nótese que este vertiginoso aumento del empleo no incidió sobre los puestos laborales de tipo estacional (Ministry of Agriculture and Food, 1993:78).

Últimamente el reclutamiento de trabajadores agrícolas se ha vuelto más difícil, pues compite con otros trabajos pesados como el de la construcción donde los salarios son más altos. Y por si fuera poco, los ingresos de los asalariados agrícolas son menores que lo que se recibe como seguro de desempleo (social welfare) ya que éste es todavía mayor en aproximadamente dos mil dólares por año que el salario agrícola equivalente. ${ }^{8}$ En otras palabras, mientras que en el conjunto de la agricultura aumentó la demanda laboral estacional, además de incrementarse en diversos productos específicos, la oferta laboral para este sector se fue haciendo más insegura debido al conjunto de factores señalados arriba.

La mayor necesidad de trabajo estacional en algunos productos tiene que ver con las características del desarrollo de los mismos a lo largo de la estación productiva ya que si no se cuenta con la mano de obra necesaria en el periodo se malogra la actividad. De ahí que a lo largo de los años y en virtud de los cambios señalados antes, poco a poco se fue haciendo más urgente la necesidad de contar con la mano de obra requerida particularmente en aquellas granjas donde es indispensable realizar diversas actividades.

\section{La solución a la escasez: iniciar programas laborales}

Los factores mencionados en el apartado anterior son los que han llevado a la Comisión de Empleo e Inmigración de Canadá a la pues-

\footnotetext{
${ }^{8}$ Información obtenida del funcionario del Ministerio de Agricultura de Ontario, quien es el representante de esa institución ante el programa de trabajadores.
} 
ta en marcha de varios programas de trabajadores. De éstos sobresale el Canadian Clearance Program con el objeto de llevar a Ontario trabajadores de otras regiones de Canadá. También destaca el programa de estudiantes para la agricultura, que canaliza estudiantes canadienses como trabajadores temporales durante las vacaciones del verano.

En otras épocas tuvieron cierta importancia el programa de estudiantes europeos de trabajadores para el tabaco que comenzó en el año 1965 y, unos años antes, en 1958, se había iniciado el programa de especialistas estadunidenses para el tabaco, etcétera.

En un documento de 1976 se lee lo siguiente:

Durante el periodo de octubre de 1974 a diciembre de 1975 los datos operativos reportaron un total de 69962 solicitudes de trabajadores hechas al Canada Farm Labour Pool Program y de este monto se realizaron 59444 referencias a los empleadores, las que resultaron finalmente en 42375 colocaciones laborales.

La fuerza laboral canadiense existente se complementó con una cantidad adicional de 7150 inmigrantes [sic] a quienes se les garantizó empleo durante la estación de la cosecha de 1975. Estos trabajadores incluyeron 5500 caribeños, 300 mexicanos y 1000 trabajadores estudiantes para el tabaco, quienes en su mayoría se destinaron a la zona suroeste de Ontario [trad. del autor]. ${ }^{9}$

Pero a pesar de los esfuerzos anteriores, estos programas no lograron mantener estable la oferta laboral particularmente en los momentos de mayor urgencia. De ahí que al paso de los años el programa de trabajadores agrícolas estacionales caribeños y mexicanos haya cobrado mayor importancia.

El programa de trabajadores agrícolas estacionales del Caribe y de México

Inicialmente este programa se diseñó más bien como un instrumento de ayuda a los países menos desarrollados y dio comienzo con la

${ }^{9}$ El programa de estudiantes europeos pasó de 947 personas en 1970, a sólo 346 en 1983 , es decir una baja de $64 \%$. Por otra parte el programa de especialistas estadunidenses del tabaco decreció en $74 \%$ en el mismo periodo. Estos datos y los inmediatos anteriores del texto aparecen en un documento titulado: "Statistical Analysis of the Canada Farm Labour Pool Program, 1976" (CFLPP, 1976). 
colaboración de Jamaica en 1966; un año después se incluyó a Trinidad y Tobago así como a Barbados y posteriormente a México en 1974, y a las islas orientales del Caribe en 1976, aunque ya dentro de un esquema de auxilio a la oferta laboral estacional de la agricultura canadiense. ${ }^{10}$ En 1966 llegaron a Ontario bajo los auspicios de este programa 264 trabajadores jamaiquinos; en 1970 este número subió a 1 113, y de ahí pasó a 4428 en 1983, pero ya incluía trabajadores de otras islas del Caribe y de México.

A lo largo de la década de los setenta, el programa experimentó fluctuaciones en cuanto al número de trabajadores hasta llegar a una cifra pico de 5912 personas en 1980, pero para 1991 el número de trabajadores de las diversas nacionalidades llegó a tener un aumento de $100 \%$ al llegar a 11907 personas (ibid.). En realidad este incremento vertiginoso, sobre todo durante la última década, es una muestra clara del éxito del programa como abastecedor parcial de la mano de obra agrícola de carácter estacional principalmente para la provincia de Ontario. ${ }^{11}$ En el cuadro 2 se presenta la información sobre el número anual de trabajadores mexicanos que ha habido a lo largo de los años desde 1974.

Pero, ¿qué proporción de la fuerza laboral estacional de la provincia está integrada por los trabajadores mexicanos y caribeños? Como veremos, los datos que existen no permiten darnos una respuesta directa, aunque a partir de las entrevistas con los encargados del programa fue posible plantear una aproximación.

Nos enfrentamos aquí a un problema donde, por un lado, hay que manejar el número de trabajadores y, por otro, el del tiempo que se trabaja. Uno de los funcionarios entrevistados en uno de los ministerios involucrados afirmó que el programa cubría entre 15 y $20 \%$ del total de la fuerza laboral estacional de la provincia. A continuación vamos a tratar de presentar un cálculo numérico aproximado basado en esa afirmación junto con algunos otros datos publicados en varios reportes.

El Agricultural Human Resources Planning Committee estimó el total de la fuerza laboral estacional para toda la provincia de Ontario en 16572 unidades equivalentes a persona-año. Por otra parte calculaba que los requerimientos estacionales de los siete cultivos que deman-

\footnotetext{
${ }^{10}$ Información proporcionada por el encargado del programa por parte del Ministerio de Recursos Humanos de Ontario.

$1195 \%$ de los trabajadores mexicanos y caribeños se orientan a granjas de la provicia de Ontario; el resto van principalmente a Quebec y a Manitoba.
} 
El PROGRAMA DE TRABAJADORES AGRICOLAS MEXICANOS CON CANADÁ 179

CUADRO 2

Evolución histórica del envío de trabajadores agrícolas a Canadá, 1974-1996

\begin{tabular}{|c|c|c|c|}
\hline \multirow[b]{2}{*}{ Años } & \multirow[b]{2}{*}{$\begin{array}{l}\text { Numero de } \\
\text { trabajadores }\end{array}$} & \multicolumn{2}{|c|}{ Cambio } \\
\hline & & Abs. & $\%$ \\
\hline 1974 & 203 & & \\
\hline 1975 & 402 & 199 & 98.0 \\
\hline 1976 & 533 & 131 & 32.6 \\
\hline 1977 & 495 & $(-38)$ & -7.1 \\
\hline 1978 & 543 & 48 & 9.7 \\
\hline 1979 & 553 & 10 & 1.8 \\
\hline 1980 & 678 & 125 & 22.6 \\
\hline 1981 & 655 & $(-23)$ & -3.4 \\
\hline 1982 & 696 & 41 & 6.3 \\
\hline 1983 & 615 & 81 & -1.6 \\
\hline 1984 & 672 & 57 & 9.3 \\
\hline 1985 & 834 & 162 & 24.1 \\
\hline 1986 & 1007 & 173 & 20.7 \\
\hline 1987 & 1538 & 531 & 52.7 \\
\hline 1988 & 2711 & 1173 & 76.3 \\
\hline 1989 & 4466 & 1755 & 64.7 \\
\hline 1990 & 5143 & 677 & 15.2 \\
\hline 1991 & 5148 & 5 & 0.1 \\
\hline 1992 & 4778 & $(-370)$ & -7.2 \\
\hline 1993 & 4872 & 94 & 2.0 \\
\hline 1994 & 4910 & 38 & 0.8 \\
\hline 1995 & 4886 & $(-24)$ & 0.4 \\
\hline 1996 & 5211 & 325 & 6.6 \\
\hline Cotal & 51549 & & \\
\hline
\end{tabular}

Fuente: STPS (1994:21; 1996).

laban más mano de obra era de 9982 unidades equivalentes a persoıa-año (60\%). Por ello, si multiplicamos esta cifra por 52 semanas, endríamos 519064 unidades persona-semana. Por otro lado, si calcuáramos que cada trabajador estacional extranjero trabaja 17 semanas n promedio, ${ }^{12}$ nos daría (si tomamos el número de 10959 trabajalores estacionales extranjeros de 1993), un total de 186303 persolas-semana, cifra que convertida a la unidad persona-año (de 52 seaanas), sería igual a 3582 unidades, o sea el equivalente a $21 \%$ de sdo el trabajo estacional de los siete cultivos.

12 Dato proporcionado por el encargado del programa por parte del Ministerio e Recursos Humanos de la provincia de Ontario. 
Sin embargo, para aquilatar la importancia verdadera del programa más que para compararlo (como se hace anterior mente, con una categoría laboral distinta como sería la del trabajo permanente durante las 52 semanas del año), habría que calibrar la incidencia del mismo en los requerimientos laborales específicos, es decir, en la satisfacción de la demanda laboral cuando más se necesita, particularmente frente a la situación real del mercado laboral agrícola estacional de algunos de los cultivos.

Quizás el problema más grave detectado por los granjeros en relación con las necesidades de trabajo estacional es el de la rotación del personal. No es tanto que el trabajador canadiense no se acerque a trabajar a las granjas, sino que no se queda cuando más se le necesita. ${ }^{13}$ En contrapartida, ésta es la mejor cualidad de los trabajadores estacionales extranjeros: llegan sólo cuando se les necesita y permanecen en las granjas todo el tiempo requerido. Se trata obviamente de una cualidad más estimada todavía cuando se trabaja con productos perecederos como los de las granjas de Ontario.

Los cultivos a los que van los trabajadores extranjeros estacionales son: frutas, verduras, tabaco, producción en invernadero, viveros, manzanas y ginseng. De éstos, el cultivo del tabaco ha estado requiriendo entre $30 \%$ y $35 \%$ de todos los trabajadores estacionales extranjeros; alrededor de $20 \%$ va a las verduras y cerca de otro tanto a las manzanas. El resto se divide en los demás cultivos (FARMS, 1994).

La mayor presencia de los extranjeros en el tabaco se debe no sólo a la necesidad de mano de obra en este cultivo, sino a la premura con que ciertas tareas se deben realizar en ese cultivo en tiempos y momentos muy determinados y para los que es indispensable contar con el número de brazos adecuado. Desde el punto de vista de la nacionalidad de los trabajadores del programa, durante los últimos años los mexicanos han constituido cerca de 37 o $38 \%$ y el resto han sido caribeños. De estos últimos, $43 \%$ han sido jamaiquinos; $8 \%$ de Trinidad y Tobago; $6 \%$ de Barbados, y $4 \%$ del Caribe oriental (FARMS, 1994).

Sin embargo, se ha ido dando cierta especialización nacional según los diversos cultivos. Así tenemos que, por ejemplo, en los traba-

13 Éste es un dato muy claro obtenido del estudio "The Monthly Demand for Labor in Ontario's Tobacco and Horticultural Industries" (Hasslett, 1986), pero la existencia de esta situación en la actualidad fue confirmada por los funcionarios del gobierno en las entrevistas que realicé con ellos. 
jos de invernadero los mexicanos llegan a ser entre 85 y $90 \%$ de todos los trabajadores del programa; en los viveros constituyen entre 65 y $75 \%$, y en la producción de verduras cerca de 55\% (FARMS, 1994). Al respecto un funcionario comentó que probablemente la mayor preferencia por los mexicanos en el caso de los cultivos de invernadero se daba porque la mayoría de los empleadores eran italianos y quizás habría una cierta "empatia latina" entre empleador y empleados.

Los caribeños, por otra parte, llegan a ser hasta $70 \%$ en el cultivo del tabaco y cerca de $80 \%$ en las huertas de manzana (FARMS, 1994).

Esta relativa especialización de los trabajadores según cultivo y nacionalidad se ha dado en parte por la costumbre que se ha desarrollado en el programa en cuanto a la solicitud de los trabajadores "por nombre", es decir, que los granjeros pueden solicitar a uno o varios trabajadores que ya han trabajado con ellos, y los trabajadores, por su parte, también pueden decidir si regresan o no con algún patrón según haya sido su experiencia. Esta práctica ha resultado en general benéfica porque ahorra tiempo de capacitación o simplemente de adaptación y en este sentido hace posible que el trabajo sea más eficaz. ${ }^{14}$

De 1991 a 1994, del total de trabajadores mexicanos, 49, 63, 61 y $59 \%$, respectivamente, habían sido trabajadores nominales (Valle, 1993; STPS, 1994) y, aunque se trata de una cifra global señala no sólo que es alto el número de trabajadores que regresa año con año, sino también que existe una relativa poca rotación anual entre las granjas, sobre todo si consideramos que se trata de trabajadores "estacionales". En los años señalados, las transferencias de trabajadores de una granja a otra en una misma temporada han fluctuado entre 4 y $7 \%$, números que nos completan la visión sobre la escasa rotación de trabajadores (Valle, 1993; STPS, 1994).

En la temporada de 1993 el rompimiento del contrato por diversas razones (médicas, familiares, etc.), alcanzó a $4 \%$ de los trabajadores mexicanos y sólo $0.5 \%$ dejó el programa por "deserción". Este último problema, sin embargo, es mayor entre los caribeños ya que abarcó a 2.5 por ciento. ${ }^{15}$

\footnotetext{
14 En el cuadro 3 se observa la situación en cuanto a los trabajadores "nominales" para 1994. Debe considerarse también que para este trabajador existe además el incentivo de que, en esos casos, el patrón es quien paga el boleto de avión de ida y vuelta.

${ }^{15}$ Información aportada por el encargado del programa por parte del Ministerio de Recursos Humanos de la provincia de Ontario.
} 


\section{CUADRO 3 \\ Trabajadores agrícolas enviados a Canadá, 1994 (por entidad federativa)}

\begin{tabular}{lrrrrr}
\hline $\begin{array}{l}\text { Entidad } \\
\text { federativa }\end{array}$ & Nominal & Selección & Sustitutos & Total & $\%$ \\
\hline Chiapas & 31 & 14 & 9 & 54 & 1.1 \\
D.F. & 104 & 68 & 60 & 232 & 4.7 \\
Guanajuato & 832 & 264 & 205 & 1301 & 26.5 \\
Guerrero & 9 & - & - & 9 & 0.2 \\
Hidalgo & 262 & 99 & 78 & 439 & 8.9 \\
Jalisco & 3 & 1 & 1 & 5 & 0.1 \\
México & 390 & 162 & 118 & 670 & 13.6 \\
Michoacán & 184 & 84 & 57 & 325 & 6.6 \\
Morelos & 232 & 101 & 53 & 386 & 7.9 \\
Nuevo León & - & 2 & - & 2 & 0.1 \\
Oaxaca & 157 & 70 & 40 & 267 & 5.4 \\
Puebla & 139 & 75 & 61 & 275 & 5.6 \\
Querétaro & 16 & - & - & 16 & 0.3 \\
San Luis Potosí & 2 & 1 & - & 3 & 0.1 \\
Tabasco & 1 & - & - & 1 & - \\
Tamaulipas & 1 & - & - & 1 & - \\
Tlaxcala & 536 & 199 & 171 & 906 & 18.5 \\
Veracruz & 3 & 7 & 2 & 12 & 0.2 \\
Yucatán & 2 & - & - & 2 & 0.1 \\
Zacatecas & 4 & - & - & 4 & 0.1 \\
Total & 2908 & 1147 & 855 & 4910 & 100.0 \\
\hline
\end{tabular}

Fuente: STPS (1994).

Los datos anteriores nos dejan ver que, a pesar de algunos problemas, el programa ha logrado estabilizarse y complementar adecuadamente las necesidades laborales regulares de los cultivos importantes de la provincia de Ontario. ${ }^{16}$ La mejor prueba de ello es que el programa lleva ya 33 años con los caribeños y 25 con los mexicanos. Otra muestra de la estabilidad del programa es que ha logrado privatizarse y seguir adelante a pesar de que ahora la carga de los gastos directos recae en los granjeros mismos. Esta privatización ocurrió en 1987.

${ }^{16}$ Estos problemas de ineficiencia son, por ejemplo, que llega entre 8 y $10 \%$ menor número de trabajadores que los que han sido requeridos, o que llegan también menos trabajadores "por nombre" que los solicitados, etcétera. 
Según los cálculos realizados por los funcionarios del gobierno canadiense vinculados directamente con el manejo del programa, la utilización estacional de la mano de obra caribeña y mexicana hace subir el costo laboral de esos trabajadores entre 16 y $25 \%$ debido a los gastos administrativos del programa, de tal manera que los granjeros se ahorrarían ese monto si sólo contrataran canadienses. ${ }^{17} \mathrm{Sin}$ embargo, como ya se vio antes, la eficiencia de estos trabajadores en función de los cultivos y, sobre todo de las circunstancias específicas del mercado laboral canadiense, ha sido sumamente adecuada de tal manera que los gastos deben de compensar los beneficios recibidos por este tipo de trabajadores, ya que de otra manera el programa no habría continuado durante tantos años.

Seguramente parte del éxito del programa ha tenido que ver con los cambios y adaptaciones que se le han hecho a lo largo del tiempo ya que, según comentarios de los funcionarios canadienses, sobre todo al inicio, hubo periodos en que se cuidó menos la selección del perfil del trabajador agrícola y ello trajo como consecuencia que hubiera más rompimientos de contratos o necesidad de transferencias entre granjas o repatriaciones antes del tiempo fijado, etc. En cambio ahora ha quedado claro que el mejor trabajador es el que ha tenido o está teniendo experiencia laboral en el campo en su país de origen.

En el cuadro 4 se presenta información sobre las entidades de origen de los trabajadores mexicanos que fueron a Canadá mediante el programa en 1994 y 1996.

Puede observarse que para 1994, en primer lugar, hubo trabajadores originarios de 20 estados del país, aunque con una relativa concentración en los estados de Guanajuato, México y Tlaxcala. Llama la atención que a excepción de Guanajuato, que tuvo a un poco más de la cuarta parte de los trabajadores, los estados que tradicionalmente han enviado más migrantes a Estados Unidos tienen proporciones bajas: Michoacán $6.6 \%$; Jalisco $0.1 \%$; Zacatecas $0.1 \%$; San Luis Potosí $0.1 \%$. En cambio los estados que tuvieron mayores proporciones en el caso canadiense fueron, después de Guanajuato, Tlaxcala con $18.5 \%$ y el Estado de México con $13.6 \%$. Por otra parte,

${ }^{17}$ Los salarios que se pagan a los trabajadores estacionales extranjeros son equivalentes a lo que se paga al mismo tipo de trabajador nacional, por ello el costo del programa hace que el costo laboral sea mayor para los granjeros cuando se contratan extranjeros. 
en términos generales, la distribución tampoco fue muy diferente para $1996 .^{18}$

CUADRO 4

Trabajadores agrícolas enviados a Canadá, 1994 y 1996 (por entidad federativa)

\begin{tabular}{lrrrc}
\hline $\begin{array}{l}\text { Entidad } \\
\text { federativa }\end{array}$ & $\begin{array}{c}1994 \\
\text { Abs. }\end{array}$ & $\%$ & $\begin{array}{c}1996 \\
\text { Abs. }\end{array}$ & $\begin{array}{c}\text { Distribución } \\
\text { relativa (\%) }\end{array}$ \\
\hline Chiapas & 54 & 1.1 & 34 & 0.6 \\
Chihuahua & - & - & 3 & 0.05 \\
D.F. & 232 & 4.7 & 205 & 3.9 \\
Guanajuato & 1301 & 26.5 & 957 & 18.5 \\
Guerrero & 9 & 0.2 & 38 & 0.7 \\
Hidalgo & 439 & 8.9 & 538 & 10.5 \\
Jalisco & 5 & 0.1 & 9 & 0.2 \\
México & 670 & 13.6 & 898 & 17.5 \\
Michoacán & 325 & 6.6 & 245 & 4.7 \\
Morelos & 386 & 7.9 & 388 & 7.5 \\
Nayarit & - & - & 1 & - \\
Nuevo León & 2 & 0.1 & - & - \\
Oaxaca & 267 & 5.4 & 182 & 7.5 \\
Puebla & 275 & 5.6 & 369 & 0.3 \\
Querétaro & 16 & 0.3 & 19 & 0.3 \\
San Luis P. & 3 & 0.1 & 15 & 0.1 \\
Tabasco & 1 & - & 4 & - \\
Tamaulipas & 1 & - & - & 23.2 \\
Tlaxcala & 906 & 18.5 & 1197 & 0.9 \\
Veracruz & 12 & 0.2 & 46 & 0.05 \\
Yucatán & 2 & 0.1 & 3 & - \\
Zacatecas & 4 & 0.1 & - & 100.0 \\
Total & 410 & 100.0 & $5151 *$ & \\
\hline
\end{tabular}

* Faltó de incluir un vuelo.

Fuente: STPS (1994; 1996).

${ }^{18}$ Aunque esta lista de los estados de origen de los trabajadores para 1994 y 1996 se presenta solamente a guisa de ejemplo, muestra, sin embargo, dos aspectos que pueden tomarse como característicos del programa: la relativa dispersión de las zonas de origen, así como la baja presencia en el programa de los estados que tradicionalmente envían migrantes a Estados Unidos. Para el año 1996 no se pudo contar con información precisa sobre los trabajadores nominales, seleccionados y sustitutos. 
De la suma de los trabajadores "nominales" y los "seleccionados" en 1994, 72\% correspondió a los primeros, circunstancia que nos deja ver la alta proporción de trabajadores que son requeridos por los empleadores mismos. Al respecto conviene recordar lo que ya mencionamos en otras páginas, que en años anteriores el número de trabajadores "por nombre" había sido también sumamente alto (entre $49 \%$ y $63 \%$ ). Además, el hecho de que cierto porcentaje de este tipo de trabajadores no llegue a su destino, ha sido una de las principales quejas, tanto por parte de los empleadores canadienses, como de los funcionarios del Ministerio de Recursos Humanos de Ontario. ${ }^{19}$ Esta solicitud de trabajadores "por nombre", es un punto de gran importancia porque los requerimientos que los empleadores hacen año con año de los mismos trabajadores son un factor central si lo vemos desde la perspectiva de "redes" en los circuitos migratorios. Hasta ahora ha sido más común enfatizar esas "redes" solamente como lazos sociales o comunitarios (que efectivamente existen en la situación estadunidense), pero no como redes laborales fomentadas por los empleadores mismos.

El caso de los trabajadores que van a Canadá deja ver cómo, a lo largo de los años, el programa laboral ha aceptado una práctica que conduce precisamente a propiciar la recurrencia de los trabajadores a la migración independientemente de la existencia de comunidades étnicas de apoyo o de parientes en el país huésped. Es decir, la recurrencia a volver con un mismo empleador; de ahí que el meollo en la formación de un proceso que lleva a que más adelante se pueda hablar de una "red", depende en buena medida de las solicitudes laborales de los empleadores. En el caso de la migración a Estados Unidos, por ejemplo, ha sido muy común escuchar de los migrantes mismos que cuando se decide el viaje de alguna persona conocida o pariente que está todavía en México, éste se hace una vez que ya se ha tenido la petición expresa del empleador que requiere del trabajador. Por ello es frecuente también que los trabajadores viajen a los mismos lugares en Estados Unidos, ya que de lo que se trata es de

19 Éste fue un comentario expresado varias veces al autor por parte de los funcionarios de ese Ministerio. Asimismo ese dato fue corroborado por los funcionarios mexicanos de la Secretaría del Trabajo, quienes explicaron que era un factor que no siempre dependía de ellos ya que es frecuente que algunos trabajadores no quieran regresar con algún empleador, o también puede suceder que a veces se dificulta la comunicación con los trabajadores en México y no se les puede hacer llegar eficientemente a sus domicilios la solicitud del empleador. 
continuar experiencias previas que han sido exitosas, laboralmente hablando, tanto para el trabajador como para el empleador..$^{20}$

\section{Reflexiones finales}

En otro nivel ha quedado pendiente una de las preguntas iniciales sobre por qué, a pesar de que el programa lleva ya tantos años, no se ha generado una corriente migratoria de mexicanos hacia Canadá si, aparentemente, están dadas las circunstancias para que ello ocurra. Ya hubo tiempo de exposición de los trabajadores al país huésped ya que es alto el porcentaje de los mismos que han regresado con el mismo empleador en dos o aun más ocasiones. Como se señalaba antes, la recurrencia a trabajar en Ontario ha facilitado el inicio de redes de relación tanto con otros trabajadores como con los empleadores, $y$, seguramente también, con base en las experiencias de algunos trabajadores, otros en sus comunidades mexicanas de origen se habrán entusiasmado para ir a Canadá, y con ello se completaría el estudio del alcance de las "redes" por lo menos en cierto nivel. Ya hubo tiempo suficiente para experimentar los beneficios del ingreso proveniente de las experiencias en Canadá; igualmente, ha pasado bastante tiempo como para que esos beneficios pudieran actuar como "efecto de demostración" en las comunidades de origen y, por ello mismo, sería lógico que mucha más gente deseara lograr una experiencia semejante.

Lo que el autor pudo indagar, tanto por medio de la consulta de reportes y estadísticas como, sobre todo, por las entrevistas con los diversos funcionarios del gobierno que están al tanto del fenómeno, es que, efectivamente, no ha existido hasta ahora ningún otro flujo migratorio legal o ilegal como efecto del programa de trabajadores. ${ }^{21}$

\footnotetext{
20 Éste es un punto en el que habría que ampliar y profundizar las investigaciones con el fin de poder determinar mejor la importancia de las relaciones propiamente laborales en la formación de redes o lazos entre personas y comunidades de una zona en México y las localidades de destino en Estados Unidos.

${ }^{21}$ Existen diversos controles en el programa tanto en México como en Canadá que permiten detectar las deserciones cuando ocurren y que permitirían saber si el programa podría ser o no un canal de paso para migrantes que después se convertirían en indocumentados. Pero tanto por las cifras de las "deserciones" que aparecen en los documentos del programa a lo largo de los años, como por la información aportada por los funcionarios de los ministerios canadienses, así como por los encargados del programa y por el consulado mexicano en Toronto y la STPS en México, hay
} 
Esta constatación resulta interesante porque permite relativizar y cuestionar algunas creencias comunes sobre la movilización de mexicanos principalmente a Estados Unidos.

Es común afirmar, por ejemplo, que la continuidad de la experiencia migratoria laboral genera migración definitiva, o que la diferencia de ingresos entre los países incentiva la migración, o que la existencia de parientes y lazos comunitarios es el factor central para aumentar las probabilidades de emigrar. Pero lo que esta experiencia con Canadá nos pone sobre la mesa es que ninguno de esos elementos son suficientes por sí mismos para generar o incentivar los movimientos migratorios indocumentados o masivos, puesto que existiendo no han dado paso al resultado esperado. Ciertamente esos factores tienen influencia e intervienen en el proceso pero no como determinantes. Seguramente la vecindad (y de ahí la cercanía) entre México y Estados Unidos facilita una mayor movilización entre los países, pero parecería también que quizás sean más determinantes o al menos tengan mucho más que ver las características de la demanda laboral junto con la instrumentación laboral y migratoria del país huésped.

Canadá, a pesar de ser un país postindustrial, tiene pocos habitantes y una economía relativamente pequeña, al menos frente a Estados Unidos. Ontario, por otra parte, tiene una economía agrícola diversificada y rica pero relativamente limitada en cuanto a las posibilidades de expansión. Por ello, aunque se amplió en $100 \%$ la demanda laboral estacional agrícola durante los últimos diez años, ello sucedió más bien por una situación de adaptación laboral del conjunto de la población nativa que por una ampliación directa de las posibilidades del sector. En otros términos, que la fuerza laboral caribeña y mexicana ha sido complementaria de la fuerza laboral agrícola canadiense. Por otra parte, la economía canadiense no ha tenido el dinamismo de California donde la ampliación de una demanda laboral diversificada ha continuado al paso de los años permitiendo que los mexicanos y asiáticos recién llegados vayan ocupando los puestos

claridad en que además de que han sido pocos los casos de deserción del programa, lo común ha sido detectar a esas personas luego en las comunidades de origen. Por otra parte, si hubieran llegado indocumentados de manera totalmente independiente del programa, su aparición y aceptación en las granjas vendría a amenazar las bases mismas de la constitución del programa de trabajadores, lo que hubiera llevado necesariamente a establecer otro tipo de controles diversos que ya tendrían que estar integrados en la mecánica del programa. 
ocupacionales más bajos mientras que otro tipo de trabajador puede cambiar de posición en la escala laboral según lo reportan, por ejemplo, Alarcón (1995) y el Estudio Binacional sobre Migración (Commission for Immigration Reform et al., 1998). Asimismo, la agricultura californiana tiene una fuerte orientación hacia la exportación de varios de sus productos, lo cual es posible además porque a lo largo de los años se ha mantenido alta la oferta laboral incidiendo así sobre el costo de la mano de obra.

En vista de la experiencia del programa de trabajadores, el autor preguntó a varios funcionarios canadienses qué tanto podría repetirse un programa de este tipo en algún otro nicho de la economía canadiense donde hubiera necesidad de trabajadores estacionales o temporales. Las respuestas fueron claras aunque no siempre convincentes.

Para alguno de ellos la inexistencia de otros programas es prueba de que no se han necesitado, aunque ello implicaría que el gobierno tuviera un papel efectivo en el reflejo de las necesidades laborales de los múltiples y diversos subsectores de la economía canadiense y esta situación no es evidente ya que supondría también contar con los instrumentos que pudieran servir para reflejar esas necesidades aun en subsectores de menor importancia económica que la producción de frutas y hortalizas.

Para algún otro funcionario, la ampliación de este tipo de programas a otros sectores implicaría problemas, al menos de imagen, pues se podría dar el mensaje de que se le están quitando posibilidades laborales a los canadienses. En parte, señalaba ese mismo funcionario, el éxito del programa de trabajadores agrícolas reside en que se orienta a un tipo de trabajos de un subsector de la economía que es muy poco ambicionado, laboralmente hablando, por el canadiense común y donde además existen grupos económicos de cierta importancia como es el caso de los productores de hortalizas y frutas.

Pero es claro que tanto para Canadá como para México el programa ha sido exitoso desde diversos puntos de vista y valdría la pena explorar la posibilidad de experiencias semejantes aunque ciertamente con el cuidado de no intentar extrapolar simplistamente las formas y mecanismos de este programa. Como se afirmaba antes, ni Canadá ni Ontario son equiparables a Estados Unidos y a California, además de que, como se vislumbra en el cuadro 2, las cifras de este programa son sumamente pequeñas comparadas con el flujo laboral hacia Estados Unidos. 
De todas maneras es estimulante saber que existe una experiencia actual de intercambio laboral entre dos países donde, además de que se reconoce la complementariedad funcional entre una oferta y una demanda laborales, ha sido posible establecer mecanismos dignos y adecuados que han permitido una saludable interacción entre los dos países.

El éxito de este programa sería suficiente para no difamar de manera generalizada a los llamados programas de trabajadores huéspedes ya que existen múltiples circunstancias que inciden en el buen o mal desarrollo de este tipo de experiencias. Por otra parte, en Estados Unidos se tuvo la experiencia del programa de trabajadores en apoyo de los ferrocarriles durante los años cuarenta, el cual fue también un programa exitoso aunque de menor duración (Driscoll, 1996).

\section{Bibliografía}

Agriculture Canada (1992), Canada's Trade in Agricultural Products, 1988, 1989 and 1990, Ottawa, Internacional Trade Policy.

Alarcón, Rafael (1995), “'Immigrants or Trasnational Workers?”, documento de trabajo, Davis, California Institute for Rural Studies.

Andarawewa, A. B. (1970), The Structure of the Canadian Agricultural Labor Force, Ottawa, Research Division, Economics Branch, Department of Agriculture.

Basran, G. S. (1992), "Changes in Agriculture in Canada: Theoretical Perspectives", en Rural Sociology in Canada, Toronto-Oxford-Nueva York, Oxford University Press.

Beach, Charles M. (ed.) (1989), Policy Forum on the Role of Immigration in Canada's Future, Kingston, John Deutsch Institute for the Study of Economic Policy, Queen's University.

Beaujot, Roderic (1988), Immigrants an Canada: Une analyse des donnees de recensement, Ottawa, Statisque Canada.

CFLPP (Canada Farm Labour Pool Program) (1976), "Statistical Analysis of the Canada Farm Labour Pool Program, 1976", documento de trabajo, CFLPP.

Commission for Immigration Reform y Secretaría de Relaciones Exteriores (1998), Estudio Binacional México-Estados Unidos sobre Migración, México, SRE.

Driscoll, Barbara (1996), Me voy pa' Pensilvania por no andar en la vagancia, México, Conaculta.

Durand, Jorge y Douglas Massey (1992), "Mexican Migration to the United States: A Critical Review”, Latin American Research Review, vol. 27, núm. 2. FARMS (1993), "Employer Information Package", Ontario, Mississauga. 
(1994), "Employer Information Package", Ontario, Mississauga.

Fisher, Lloyd H. (1953), The Harvest Labor Market, Cambrigde, Harvard University Press.

Foot, David K. (1987), "Population Aging and the Canadian Labour Force", Institute for Reseach on Public Policy (documento de trabajo 87.A.5).

Hammar, Tomas (ed.) (1985), European Immigration Policy. A Comparative Study, Cambridge, Cambridge University Press.

Hasslett, Earl (1986), "The Monthly Demand for Labor in Ontario's Tobacco and Horticultural Industries", reporte preparado para el Joint Ontario Agricultural Human Resources Planning Committee, abril de 1985, revisado en febrero de 1986 (mimeo.).

Hay, David A. (1992), "Rural Canada in Transition: Trends and Developments", en David Hay y Gurcharn S. Basran (eds.), Rural Sociology in Canada, Toronto-Oxford-Nueva York, Oxford University Press.

Joint Ontario Agricultural Human Resources Planning Committee (1986), "Seasonal Labor in the Ontario Tobacco and Horticultural Industries", reporte de trabajo, junio (mimeo.).

Kissam, Edward, Ana García y David Runsten (1992), "The Apple and Asparagus Industries in Washington", en Commission on Agricultural Workers, 1989-1993, apéndice I, Washington, noviembre (Case Studies and Research Reports).

Knowles, Valerie (1992), Strangers at our Gates, Canadian Immigration Policy, 1540-1990, Toronto-Oxford, Dondurn Press.

Massey, Douglas, K. Espinosa y J. Durand (1995), "Dinámica migratoria entre México y Estados Unidos", ponencia presentada en la V: Reunión Nacional de Investigación Demográfica en México, junio, México (mimeo.).

Ministry of Agriculture and Food (1991), 1990, Agricultural Statistics for Ontario, Queen's Printer for Ontario.

(1993), 1992, Agricultural Statistics for Ontario, Queen's Printer for Ontario.

Norrie, Kenneth y Douglas Owram (1991), A History of the Canadian Economy, Toronto, Harcourt Brace Jovanovich.

Pérk. z, Josafat (1989), "Diagnóstico de análisis sobre el programa de trabajadores migratorios a Canadá", México, STPS (mimeo.).

Simmons, Alan (1993), "Latin American Migration to Canada: New Linkages in the Hemispheric Migration and Refugee Flow System", International Journal, vol. 48, primavera.

Statistics Canada (1992), Agricultural Profile of Ontario, parte 2, diciembre, Household Surveys Division. (1993), Labour Force Annual Averages, Household Surveys Division.

STPS (1994), "Evaluación del Programa de Trabajadores Agrícolas Migratorios Temporales Mexicanos con Canadá 1989-1994”, México, STPS, Dirección General de Empleo (mimeo.). 
(1996), "Reporte sobre número de trabajadores mexicanos que van a Canadá, 1996", México, STPS (mimeo.).

United Nations Economic Commission for Europe (1994), International Migration, Regional Processes and Responses, Nueva York-Ginebra (Economic Studies, 7).

Valle Cárdenas, Enrique (1993), "Evaluación del Programa de Trabajadores Agrícolas Temporales, 1993", Toronto, Consulado General de México en Toronto (mimeo.).

Verduzco Igartúa, Gustavo (1995), "La migración mexicana a Estados Unidos: recuento de un proceso histórico", Estudios Sociológicos, vol. 13, núm. 39.

Vogt, Ruy, Beverly Cameron y Edwin Dolan (1993), Economics. Understanding the Canadian Economy, Toronto, Holt Reinhart and Winston.

Wall, Ellen (1994), "Farm Labour Markets and the Structure of Agriculture", The Canadian Review of Sociology and Anthropology, vol. 31, núm. 1. 
